\title{
Identification of Intestinal UDP-Glucuronosyltransferase Inhibitors in Green Tea (Camellia sinensis) Using a Biochemometric Approach: Application to Raloxifene as a Test Drug via In Vitro to In Vivo Extrapolation ${ }^{\mathbb{\$}}$
}

\author{
Dan-Dan Tian, Joshua J. Kellogg, Neşe Okut, Nicholas H. Oberlies, Nadja B. Cech, \\ Danny D. Shen, Jeannine S. McCune, and Mary F. Paine
}

Department of Pharmaceutical Sciences, College of Pharmacy, Washington State University, Spokane, Washington (D.-D.T., M.F.P.); Department of Chemistry and Biochemistry, University of North Carolina at Greensboro, Greensboro, North Carolina (J.J.K., N.O., N.H.O., N.B.C.); Department of Pharmaceutics, School of Pharmacy, University of Washington, Seattle, Washington (D.D.S.); and Department of Population Sciences, City of Hope, Duarte, California (J.S.M.)

Received November 16, 2017; accepted February 14, 2018

\begin{abstract}
Green tea (Camellia sinensis) is a popular beverage worldwide, raising concern for adverse interactions when co-consumed with conventional drugs. Like many botanical natural products, green tea contains numerous polyphenolic constituents that undergo extensive glucuronidation. As such, the UDP-glucuronosyltransferases (UGTs), particularly intestinal UGTs, represent potential first-pass targets for green tea-drug interactions. Candidate intestinal UGT inhibitors were identified using a biochemometrics approach, which combines bioassay and chemometric data. Extracts and fractions prepared from four widely consumed teas were screened (20-180 $\mu \mathrm{g} / \mathrm{ml}$ ) as inhibitors of UGT activity (4-methylumbelliferone glucuronidation) in human intestinal microsomes; all demonstrated concentration-dependent inhibition. A biochemometrics-identified fraction rich in UGT inhibitors from a representative tea was purified further and subjected to second-stage biochemometric analysis.
\end{abstract}

Five catechins were identified as major constituents in the bioactive subfractions and prioritized for further evaluation. Of these catechins, (-)-epicatechin gallate and (-)-epigallocatechin gallate showed concentration-dependent inhibition, with $\mathbf{I C}_{50}$ values (105 and $59 \mu \mathrm{M}$, respectively) near or below concentrations measured in a cup $(240 \mathrm{ml})$ of tea ( 66 and $240 \mu \mathrm{M}$, respectively). Using the clinical intestinal UGT substrate raloxifene, the $K_{\mathrm{i}}$ values were $\sim 1.0$ and $2.0 \mu \mathrm{M}$, respectively. Using estimated intestinal lumen and enterocyte inhibitor concentrations, a mechanistic static model predicted green tea to increase the raloxifene plasma area under the curve up to 6.1- and 1.3-fold, respectively. Application of this novel approach, which combines biochemometrics with in vitro-in vivo extrapolation, to other natural product-drug combinations will refine these procedures, informing the need for further evaluation via dynamic modeling and clinical testing.
Introduction

Infusions made from leaves of the plant Camelia sinensis (L.) Kuntze (Theaceae), otherwise known as green tea, are some of the most commonly consumed beverages worldwide. Green tea represents approximately $35 \%$ of total tea production (http://www.fao.org/3/ a-i4480e.pdf). Green tea supplements also have increased in popularity, ranking fourth in sales of herbal/botanical products in the United States in 2016 (Smith et al., 2017). Green tea products are promoted extensively for cardioprotection, chemoprevention, and weight loss (Moore et al., 2009; Deka and Vita, 2011; Yang and Wang, 2011). These

This project was supported by the National Institutes of Health National Center for Complimentary and Integrative Health, specifically the Center of Excellence for Natural Product Drug Interaction Research [Grant U54 AT008909], and the National Institutes of Health National Institute of General Medical Sciences [Grant R01 GM077482].

https://doi.org/10.1124/dmd.117.079491

SThis article has supplemental material available at dmd.aspetjournals.org. properties have been attributed to polyphenols known as catechins (Fig. 1), which represent major constituents of the tea beverage and are well-studied phytochemicals (Balentine et al., 1997; Schönthal, 2011; Yang and Pan, 2012). The increasing popularity of green tea products, particularly in the United States (Smith et al., 2017), increases the likelihood of co-consumption with conventional medications, which can lead to alterations in drug disposition, potentially compromising drug safety and efficacy (Brantley et al., 2014).

Clinical pharmacokinetic green tea-drug interaction studies involving various green tea products as precipitants have focused primarily on the cytochrome P450s and transport proteins as targets (Donovan et al., 2004; Chow et al., 2006; Misaka et al., 2014). Results from these studies indicated minimal to no effects on the pharmacokinetics of probe substrates for CYP1A2 (caffeine), CYP2C9 (losartan), CYP2D6 (dextromethorphan), and CYP3A4 (alprazolam and buspirone) when coadministered with green tea as capsules, extracts, or beverages. In contrast, a canned green tea beverage significantly decreased the area under the concentration-time curve (AUC) of the beta blocker nadolol

ABBREVIATIONS: AUC, area under the concentration-time curve; EC, (-)-epicatechin; ECG, (-)-epicatechin gallate; EGC, (-)-epigallocatechin; EGCG, (-)-epigallocatechin gallate; HIM, human intestinal microsome; MS, mass spectrometry; 4-MU, 4-methylumbelliferone; PLS, partial least squares; UGT, UDP-glucuronosyltransferase. 
(C)

$(+)$-catechin<smiles>Oc1cc(O)c2c(c1)O[C@H](c1ccc(O)c(O)c1)[C@H](O)C2</smiles>

(-)-epicatechin (EC)<smiles>Oc1cc(O)c2c(c1)O[C@H](c1cc(O)c(O)c(O)c1)[C@H](O)C2</smiles>

(-)-epigallocatechin (EGC)<smiles>O=C(O[C@H]1Cc2c(O)cc(O)cc2O[C@H]1c1ccc(O)c(O)c1)c1cc(O)c(O)c(O)c1</smiles>

(-)-epicatechin gallate (ECG)<smiles>O=C(O[C@H]1Cc2c(O)cc(O)cc2O[C@H]1c1cc(O)c(O)c(O)c1)c1cc(O)c(O)c(O)c1</smiles>

(-)-epigallocatechin gallate (EGCG)
Fig. 1. Structures of (+)-catechin, EC, EGC, ECG, and EGCG (by $85 \%$ relative to baseline), which was accompanied by an attenuated decrease (approximately 12\%) in systolic blood pressure. The pharmacokinetic interaction was attributed to inhibition of an apically located uptake transporter in the intestine by green tea, specifically OATP1A2 (Misaka et al., 2014). Although the existence of intestinal OATP1A2 is controversial (Glaeser et al., 2007; Drozdzik et al., 2014), these observations, combined with the well-known effects of grapefruit juice and other fruit juices on intestinal CYP3A4 and/or OATP (Won et al., 2012; Yu et al., 2017), further highlight intestinal enzymes/transporters as key targets for natural product-drug interactions.

Compared with the cytochrome P450s and transporters (Muto et al., 2001; Donovan et al., 2004; Chow et al., 2006; Misaka et al., 2013, 2016; Mooiman et al., 2014; Knop et al., 2015), the effects of green tea and constituents on other biochemical targets are understudied. Like other botanical/natural products, green tea is rich in polyphenolic constituents that undergo extensive phase II conjugation (i.e., sulfation and/or glucuronidation) upon oral administration (Lu et al., 2003a; Sang et al., 2011). As such, these constituents represent potential inhibitors of phase II enzymes. Previous studies reported (-)-epigallocatechin gallate (EGCG) to inhibit UDP-glucuronosyltransferases (UGTs) using mouse and human liver microsomes, with $\mathrm{IC}_{50}$ values $\geq 17 \mu \mathrm{M}$ (Lu et al., 2003a; Mohamed et al., 2010; Mohamed and Frye, 2011; Jenkinson et al., 2012), which greatly exceed maximum plasma concentrations reported in humans $(<5 \mu \mathrm{M})$ (Nakagawa et al., 1997; Chow et al., 2001, 2003; Renouf et al., 2013; Misaka et al., 2014). However, these observations do not rule out an interaction in the intestine due to higher constituent concentrations and well-known differences in the UGT milieu between the liver and intestine (Uchaipichat et al., 2004; Gufford et al., 2014, 2015b). Intestinal UGTs contribute substantively to the firstpass metabolism of several orally administered drugs, including the immunosuppressant mycophenolic acid, the antihyperlipidemic agent ezetimibe, and the antiosteoporosis agent raloxifene. Thus, intestinal UGTs were prioritized as potential targets for green tea as a precipitant of drug interactions.

Botanical natural products, including green tea, are complex and variable mixtures of diverse phytoconstituents. Comprehensive, robust methods are needed to identify candidate precipitants of interactions with drugs. Bioassay-guided fractionation is an iterative approach that uses bioassay information to inform multiple stages of chromatographic separation and isolation of putatively active constituents. Bioassay-guided fractionation remains a primary approach for identifying bioactive constituents in complex botanical mixtures (Ngo et al., 2009; Kim et al., 2011; Roth et al., 2011). Biochemometrics, which combine bioassay data with mass spectrometry (MS)-generated metabolomics data, represent a more contemporary, comprehensive method for identifying and prioritizing bioactive constituents from natural products (Inui et al., 2012; Kellogg et al., 2016). This approach was used successfully to identify antimicrobial constituents in fungi (Kellogg et al., 2016) and goldenseal (Britton et al., 2017) but has not yet been applied to the identification of inhibitors of drug metabolizing enzymes.

The objective of the current work was to identify candidate intestinal UGT inhibitors in green tea that could precipitate a clinical pharmacokinetic green tea-drug interaction. The aims were to 1) identify potential intestinal UGT inhibitors using a biochemometrics approach and prioritize for further evaluation, 2) determine the inhibition kinetics of prioritized constituents using raloxifene as a clinical intestinal UGT substrate, and 3) evaluate the potential for a green tea-raloxifene interaction in vivo. This systematic approach could be applied to other natural products with the potential to precipitate pharmacokinetic interactions with conventional drugs.

\section{Materials and Methods}

Materials and Chemicals. Human intestinal microsomes (HIMs) (pooled from 10 donors, mixed gender, lot no. 1410074) were purchased from XenoTech, LLC (Lenexa, KS). EGCG and L-ascorbic acid were purchased from Cayman Chemical Company (Ann Arbor, MI). Raloxifene was purchased from BIOTANG Inc. (Lexington, MA). (+)-Catechin, (-)-epicatechin gallate (ECG), (-)-epicatechin (EC), ethyl gallate, raloxifene-4'-glucuronide, and of raloxifene6-glucuronide were purchased from Toronto Research Chemicals Inc. (Toronto, Ontario, Canada). Alamethicin, bovine serum albumin, caffeine, (-)-epigallocatechin (EGC), magnesium chloride, 4-methylumbelliferone (4-MU), nicardipine, saccharolactone, naringin, and UDP-glucuronic acid were purchased from SigmaAldrich (St. Louis, MO). Silybin B was purified from silymarin (Euromed S.A., Barcelona, Spain) as previously described (Graf et al., 2007). Methanol (liquid chromatography MS grade), formic acid, Tris base, and Tris- $\mathrm{HCl}$ were purchased from Fisher Scientific (Waltham, MA).

Biochemometrics of Green Tea to Identify Intestinal UGT Inhibitors. Four representative, bagged green teas, coded T02, T07, T13, and T21 (described in detail in Kellogg et al., 2017), were selected for testing as intestinal UGT inhibitors (Fig. 2). Standard reference material of green tea leaves (no. 3254) was obtained from the National Institute of Standards and Technology (coded T26). 


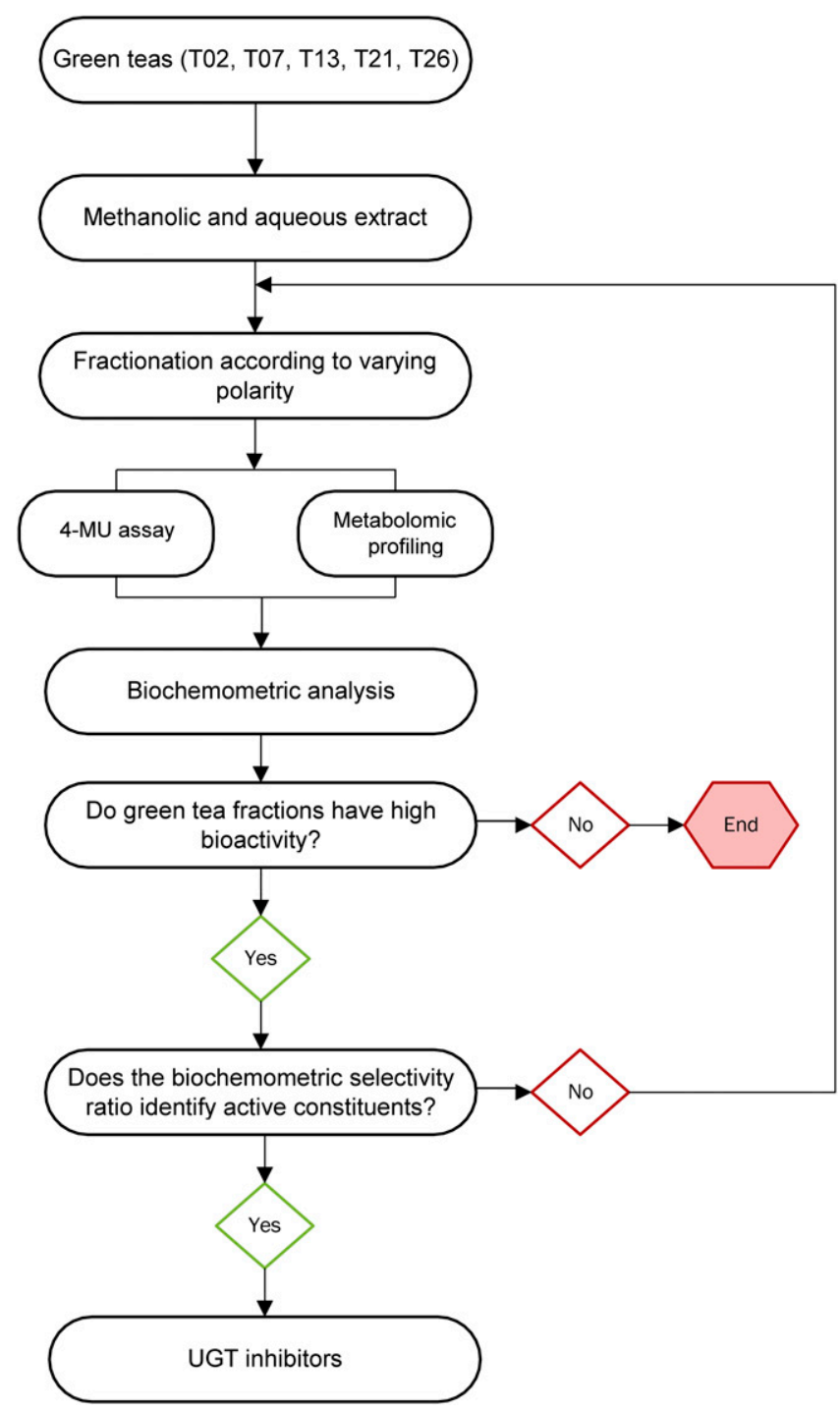

Fig. 2. Workflow for biochemometrics-guided fractionation of green teas to identify candidate intestinal UGT inhibitors.

Extracts of these five teas were prepared as previously described (Kellogg et al., 2017). Briefly, methanol (20 ml) was added to scintillation vials containing $200 \mathrm{mg}$ of dried leaves. After shaking overnight at room temperature, the contents were filtered and dried under nitrogen. The extracts were submitted to first-stage fractionation using normal-phase flash chromatography with a CombiFlash RF system with a 4-g silica gel column (Teledyne-Isco, Lincoln, NE). The solvent consisted of hexane:chloroform:methanol with the following gradient (flow rate, $18 \mathrm{ml} / \mathrm{min}$ ): 0-9.5 minutes, $100: 0: 0$ to $0: 100: 0 ; 9.5-18$ minutes, $0: 100: 0 ; 18$ 32.4 minutes, $0: 100: 0$ to $0: 80: 20 ; 32.4-37.6$ minutes, $0: 80: 20 ; 37.6-42.8$ minutes, $0: 80: 20$ to $0: 50: 50 ; 42.8-48.0$ minutes, $0: 50: 50 ; 48.0-53.2$ minutes, $0: 50: 50$ to 0 : $0: 100$; and 53.2-58.4 minutes, 0:0:100. Fractions were collected every 30 seconds for 60 minutes and pooled based on UV chromatograms $(\lambda, 280 \mathrm{~nm})$, yielding five pooled fractions (A-E); these pools were dried under nitrogen. Fractions of a hot water extract of T26 were prepared similarly. All extracts and fractions were stored dry at $4{ }^{\circ} \mathrm{C}$ to prevent degradation of catechins and other constituents, an approach used routinely to maintain stability of natural product constituents; commercially available standards of the major constituents in green tea (catechins) were stable under these same storage conditions. The five fractions and original extract from each tea were tested as inhibitors of intestinal 4-MU glucuronidation (vide infra).

Untargeted metabolomic data for extracts and fractions of the green teas were acquired as previously described (Kellogg et al., 2017) using a Q Exactive Plus quadrupole-orbitrap mass spectrometer (Thermo Scientific, Bellefonte, PA) with an electrospray ionization source (operated in a switching positive/negative mode) coupled to an Acquity UPLC System (Waters, Milford, MA). Briefly, each extract and fraction was resuspended in methanol $(1 \mathrm{mg} / \mathrm{ml})$, and a $3-\mu l$ aliquot was injected onto an Acquity UPLC BEH C18 column $(1.7 \mu \mathrm{m}, 2.1 \times 50 \mathrm{~mm})$. The following binary gradient $(0.3 \mathrm{ml} / \mathrm{min})$ consisting of water (A) and acetonitrile (B), both of which contained $0.1 \%$ formic acid, was applied: $0-1.0$ minutes, $5 \% \mathrm{~B}$; $1.0-12.0$ minutes, $5 \%-100 \% \mathrm{~B} ; 12.0-14.0$ minutes, $100 \% \mathrm{~B} ; 14.0-16.0$ minutes, $100 \%-5 \% \mathrm{~B}$; and $16.0-17.0$ minutes, $5 \% \mathrm{~B}$. A mixture containing catechins (EC, ECG, EGC, and EGCG) and caffeine was injected every five runs as an internal quality control measure.

The metabolomics data sets for each extract and fraction were analyzed, aligned, and filtered using MZmine 2.25 software (Pluskal et al., 2010) with parameter settings as previously described (Kellogg et al., 2017). The spectral data matrix (consisting of $\mathrm{m} / \mathrm{z}$, retention time, and peak area) was imported to Excel (Microsoft, Redmond, WA) and merged with the bioactivity data (at the $60 \mu \mathrm{g}$ extract/ml concentration) to form a final biochemometric analytical matrix. Data sets for each extract/fraction consisted of triplicate bioassay measurements and triplicate high-resolution MS analyses. Biochemometric analysis was conducted using Sirius (version 10.0; Pattern Recognition Systems AS, Bergen, Norway) (Kvalheim et al., 2011; Kellogg et al., 2016) after a fourth root transformation of the spectral variables to reduce heteroscedasticity. An unsupervised statistical analysis was achieved using principal component analysis. An internally crossvalidated four-component partial least-squares (PLS) model was constructed using 100 iterations at a significance level of 0.05 . Selectivity ratios from the final PLS model were calculated using algorithms internal to Sirius.

Based on the biochemometric analysis, fraction $\mathrm{C}$ from a representative tea (T21) was advanced to second-stage purification (Fig. 2) using a reverse-phase preparative high-performance LC with a Gemini NX C18 column $(5 \mu \mathrm{m}, 250 \times$ $21.2 \mathrm{~mm}$; Phenomenex, Torrance, CA). Using a linear gradient from 60:40 water: acetonitrile (each containing $0.1 \%$ formic acid) to $100 \%$ acetonitrile, fractions were collected every 0.5 minutes for 15 minutes and were pooled based on UV chromatograms, yielding eight subfractions $(\mathrm{C} 1-\mathrm{C} 8)$. These subfractions were tested as intestinal UGT inhibitors and analyzed via biochemometrics to identify UGT inhibitory constituents.

Screening of Green Tea Fractions, Subfractions, and Purified Catechins as Inhibitors of Intestinal 4-MU Glucuronidation. Green tea extracts, fractions, subfractions, and purified catechins [(+)-catechin, EC, EGC, ECG, and EGCG] (Fig. 1) were screened as inhibitors of 4-MU intestinal glucuronidation as previously described (Gufford et al., 2014) with modifications. In brief, incubation mixtures consisted of HIMs $(0.2 \mathrm{mg} / \mathrm{ml})$; bovine serum albumin $(0.05 \%)$; Tris- $\mathrm{HCl}$ buffer $(\mathrm{pH} 7.4)$ supplemented with magnesium chloride (5 mM); saccharolactone $(100 \mu \mathrm{M})$; alamethicin $(50 \mu \mathrm{g} / \mathrm{mg}$ protein); 4-MU $(100 \mu \mathrm{M})$; the positive control UGT inhibitors nicardipine $(400 \mu \mathrm{M})$ or silybin B $(100 \mu \mathrm{M})$ (Gufford et al., 2014); and green tea extract/(sub)fraction (20, 60, and $180 \mu \mathrm{g} / \mathrm{ml})$ or purified catechin $(100 \mu \mathrm{M})$. Ascorbic acid $(300 \mu \mathrm{M})(\mathrm{Lu}$ et al., 2003b) was added to stabilize the catechins. The final solvent (methanol) concentration was $2 \%(\mathrm{v} / \mathrm{v})$. After equilibrating the mixtures for 10 minutes at $37^{\circ} \mathrm{C}$, reactions were initiated by adding UDP-glucuronic acid $(2 \mathrm{mM}) .4-\mathrm{MU}$ depletion (glucuronidation) was monitored via fluorescence (excitation wavelength, $365 \mathrm{~nm}$; emission wavelength, $450 \mathrm{~nm}$ ) using a Synergy H1M monochromator-based multimode microplate reader (BioTek, Winooski, VT). Velocities, determined by the slopes of the 4-MU concentration versus time data during the linear phase, were expressed as percentage of control activity.

The $\mathrm{IC}_{50}$ values for ECG and EGCG toward 4-MU glucuronidation were determined using identical conditions except that ECG and EGCG concentrations ranged from 3.9-800 to 3.9-600 $\mu \mathrm{M}$, respectively. Initial estimates of $\mathrm{IC}_{50}$ were obtained from plots of the velocity of 4-MU depletion versus the natural logarithm of inhibitor concentration. Final estimates were obtained by fitting the standard equation to the data, with and without the Hill coefficient, using Phoenix WinNonlin (version 6.4; Certara, Princeton, NJ) as previously described (Gufford et al., 2014).

Determination of the $K_{\mathrm{i}}$ Values for ECG and EGCG toward the Intestinal Glucuronidation of Raloxifene. The $K_{\mathrm{i}}$ values for ECG and EGCG were determined using raloxifene as a clinically used substrate and a $6 \times 6$ matrix of substrate and inhibitor concentrations. Incubation mixtures emulated those described for the 4-MU assay except that the final concentration of HIMs was $0.05 \mathrm{mg} / \mathrm{ml}$, raloxifene concentrations ranged from 0.25 to $10 \mu \mathrm{M}$, ECG ranged from 0.5 to $8 \mu \mathrm{M}$, and EGCG ranged from 1 to $16 \mu \mathrm{M}$. Reactions were terminated 
TABLE 1

Input properties for simulating average maximum ECG and EGCG concentrations in enterocytes using Simcyp

\begin{tabular}{lcc}
\hline \multicolumn{1}{c}{ Parameter } & ECG & EGCG \\
\hline Molecular weight & 442.4 & 458.4 \\
Log P $^{a}$ & 2.2 & 1.81 \\
$\mathrm{p} K_{\mathrm{a}}^{a}$ & $7.8,9.5$ & $7.8,9.0$ \\
$\mathrm{Blood}_{\mathrm{p}}{ }_{b}$ & 1 & 1 \\
$\mathrm{f}_{\mathrm{u}, \mathrm{plasma}}$ & 0.12 & 0.16 \\
$\mathrm{P}_{\text {eff }}\left(10^{-4} \mathrm{~cm} / \mathrm{s}\right)^{c}$ & 0.29 & 0.32 \\
$V_{\text {ss }}(1 / \mathrm{kg})^{d}$ & 0.66 & 0.42 \\
$\mathrm{CL}_{\mathrm{p} . \mathrm{o}}(\mathrm{l} / \mathrm{h})^{e}$ & 567 & 690 \\
\hline
\end{tabular}

${ }^{a}$ Predicted from physicochemical properties using ACD I-Laboratory 2.0 (v12.1.0.50375; Toronto, Ontario, Canada).

${ }^{b}$ Fraction unbound in plasma; predicted using the QSAR method within Simcyp.

${ }^{c}$ Human jejunum effective permeability; predicted using Simcyp apparent permeability $\left(\mathrm{P}_{\text {app }}\right)$ values obtained from the literature (Vaidyanathan and Walle, 2003; Song et al., 2014).

${ }^{d}$ Volume of distribution at steady state; predicted using the Rodgers and Rowland method within Simcyp.

${ }^{e}$ Apparent oral clearance; estimated by the ratio of oral dose to area under the plasmaconcentration-time curve obtained from the literature (Misaka et al., 2014).

after 4 minutes by adding ice-cold methanol $(200 \mu \mathrm{l})$ containing naringin $(1 \mu \mathrm{M})$ as the internal standard. After centrifugation, the supernatant $(3 \mu \mathrm{l})$ was injected into a 6500 QTRAP mass spectrometer (AB Sciex, Framingham, MA) interfaced to a Shimadzu LC-30AD UPLC (Shimadzu Corporation, Tokyo, Japan).

The primary glucuronides of raloxifene, raloxifene- 4 '-glucuronide, and raloxifene-6-glucuronide were quantified as previously described (Gufford et al., 2015b) with modifications. In brief, chromatographic separation of the analytes was achieved using an AQUASIL C18 column $(3 \mu \mathrm{m}, 2.1 \times 50 \mathrm{~mm}$; Thermo Scientific) and a mobile phase consisting of water (A) and methanol (B), both containing $0.1 \%$ formic acid. The following gradient $(0.5 \mathrm{ml} / \mathrm{min})$ was applied: 0-0.5 minutes, $10 \% \mathrm{~B}$; 0.5-2.0 minutes, $10 \%-45 \% \mathrm{~B} ; 2.0-3.0$ minutes, $45 \%$ B; 4.1 minutes, $45 \%-90 \%$ B; and 4.1-5.0 minutes, $10 \%$ B. Analytes were quantified using the ion transitions $650.0 \rightarrow 474.0$ (raloxifene- 4 ' -glucuronide and raloxifene-6-glucuronide) in positive mode and $579.0 \rightarrow 271.0$ (naringin) in negative mode. Data were acquired and analyzed using Analyst (version 1.6.2; AB Sciex).

Initial estimates of the $K_{\mathrm{m}}$ and $V_{\max }$ values were obtained from Eadie-Hofstee plots; initial estimates of the $K_{\mathrm{i}}$ values were obtained from Dixon plots. Final parameters were obtained by fitting the competitive, uncompetitive, and mixed inhibition models to the data via nonlinear least-squares regression using Phoenix WinNonlin as previously described (Gufford et al., 2015b).

Quantification of Catechins in T21 Tea. A cup of hot tea was prepared from $\mathrm{T} 21$ and analyzed for catechins to compare with $\mathrm{IC}_{50}$ values and estimate the dose of catechin for the in vitro-in vivo prediction (vide infra). One teabag was steeped with $240 \mathrm{ml}$ of hot water at $80^{\circ} \mathrm{C}$ for 3 minutes, and the bag was squeezed over the liquid, which was cooled at room temperature to $50^{\circ} \mathrm{C}$. A $1-\mathrm{ml}$ aliquot was removed, to which $20 \mu \mathrm{l}$ ascorbic acid $(20 \%$, w/w) was added to stabilize the catechins. Concentrations of (+)-catechin, EC, EGC, ECG, and EGCG were measured in triplicate using the same gradient as that for the raloxifene and raloxifene glucuronide measurement. The 6500 QTRAP mass spectrometer was applied in negative mode with the following ion transitions $289.0 \rightarrow 245.0$ [(+)-catechin and EC], 305.0 $\rightarrow 124.9$ (EGC), $441.0 \rightarrow 169.0$ (ECG), $457.0 \rightarrow$ 168.9 (EGCG), and 197.0 $\rightarrow 123.9$ (ethyl gallate; internal standard). The linear range for all catechins was $20.6-5000 \mathrm{nM}$. All calibration standards and quality controls were judged for batch quality based on the U.S. Food and Drug Administration guidance for industry regarding bioanalytical method validation (Food and Drug Administration Center for Drug Evaluation and Research, 2013).

In Vitro-In Vivo Prediction of a Green Tea-Raloxifene Interaction. A mechanistic static model (Fahmi et al., 2009) was used to predict the change in raloxifene AUC in the presence of ECG or EGCG:

$$
\frac{\mathrm{AUC}_{\mathrm{i}}}{\mathrm{AUC}}=\frac{1}{\left(1-\mathrm{F}_{\mathrm{g}}\right) \times\left\{1+\left[\left(\mathrm{I}_{\mathrm{g}} \times \mathrm{f}_{\mathrm{u}, \mathrm{g}}\right) /\left(K_{i} \times \mathrm{f}_{\mathrm{u}, \mathrm{mic}}\right)\right]\right\}+\mathrm{F}_{\mathrm{g}}}
$$

where $\mathrm{AUC}_{\mathrm{i}}$ is the $\mathrm{AUC}$ of the object drug (raloxifene) in the presence of inhibitor; $\mathrm{F}_{\mathrm{g}}$ is the fraction of the object drug that escapes intestinal extraction (0.054 for raloxifene) (Mizuma, 2009); $\mathrm{f}_{\mathrm{u}, \mathrm{g}}$ is the unbound fraction of the inhibitor in the gut; and $\mathrm{f}_{\mathrm{u}, \mathrm{mic}}$ is the unbound fraction of the inhibitor in HIMs. The fraction of raloxifene metabolized by UGTs in the intestine was set at 1 because glucuronidation was estimated to contribute up to $97 \%$ of total intestinal clearance (Cubitt et al., 2011). $I_{\mathrm{g}}$ is the inhibitor concentration in the intestine, which was calculated using two methods. Method 1 used the following standard equation (Rostami-Hodjegan and Tucker, 2004):

$$
\mathrm{I}_{\mathrm{g}}=\frac{\mathrm{F}_{\mathrm{a}} \times k_{\mathrm{a}} \times \text { Dose }}{\mathrm{Q}_{\mathrm{ent}}}
$$

where $F_{a}$ is the fraction of the oral dose absorbed into enterocytes, which was estimated for ECG and EGCG using Simcyp (version 15.1; SimCYP, Sheffield, United Kingdom); $k_{\mathrm{a}}$ is the first-order absorption rate constant, which was set at the default value of 0.1 minute $^{-1}$ (Ito et al., 1998); and $Q_{\text {ent }}$ is blood flow through
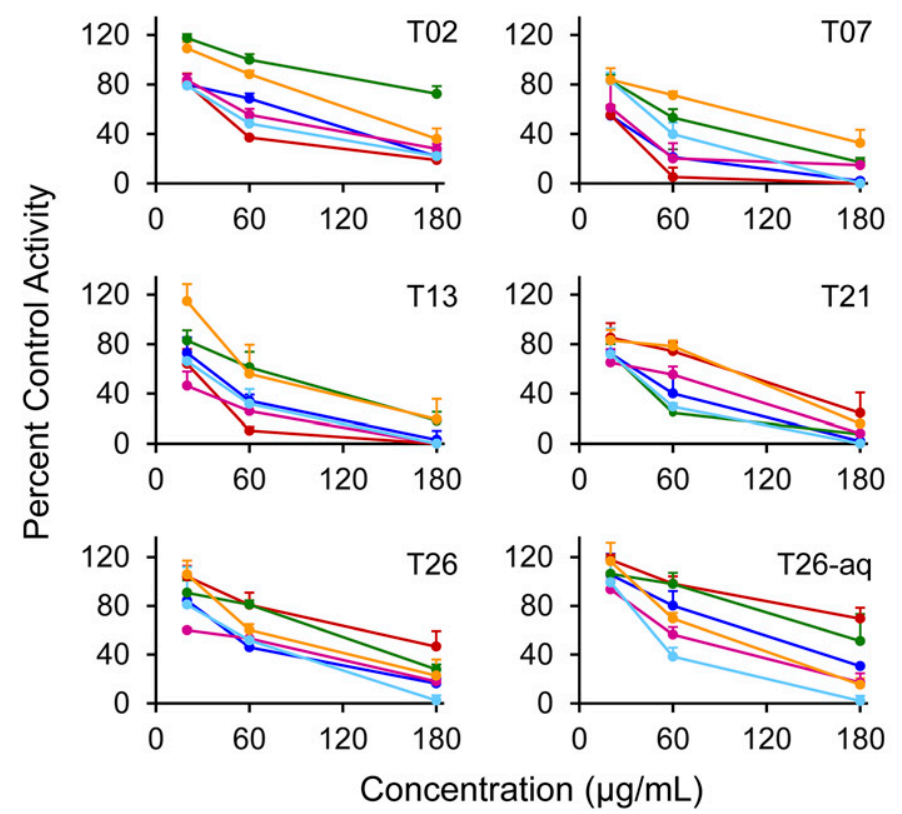

Fig. 3. Concentration-dependent inhibition of intestinal microsomal UGT activity (4-MU glucuronidation) by green tea extracts prepared from four commercially available green teas (coded T02, T07, T13, and T21) and the National Institute of Standards and Technology reference material (T26) and corresponding fractions. Symbols and error bars denote mean and S.D., respectively, of triplicate incubations. T26-aq, aqueous extract. Control activity was $4.7 \pm 0.75 \mathrm{nmol} / \mathrm{min} / \mathrm{mg}$. 


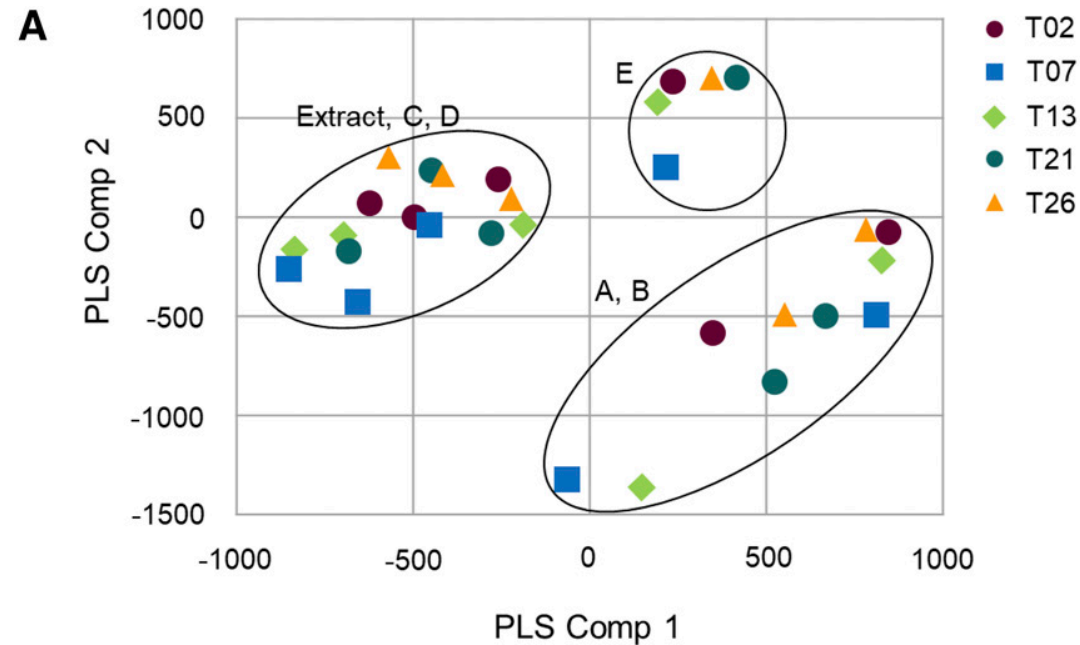

B

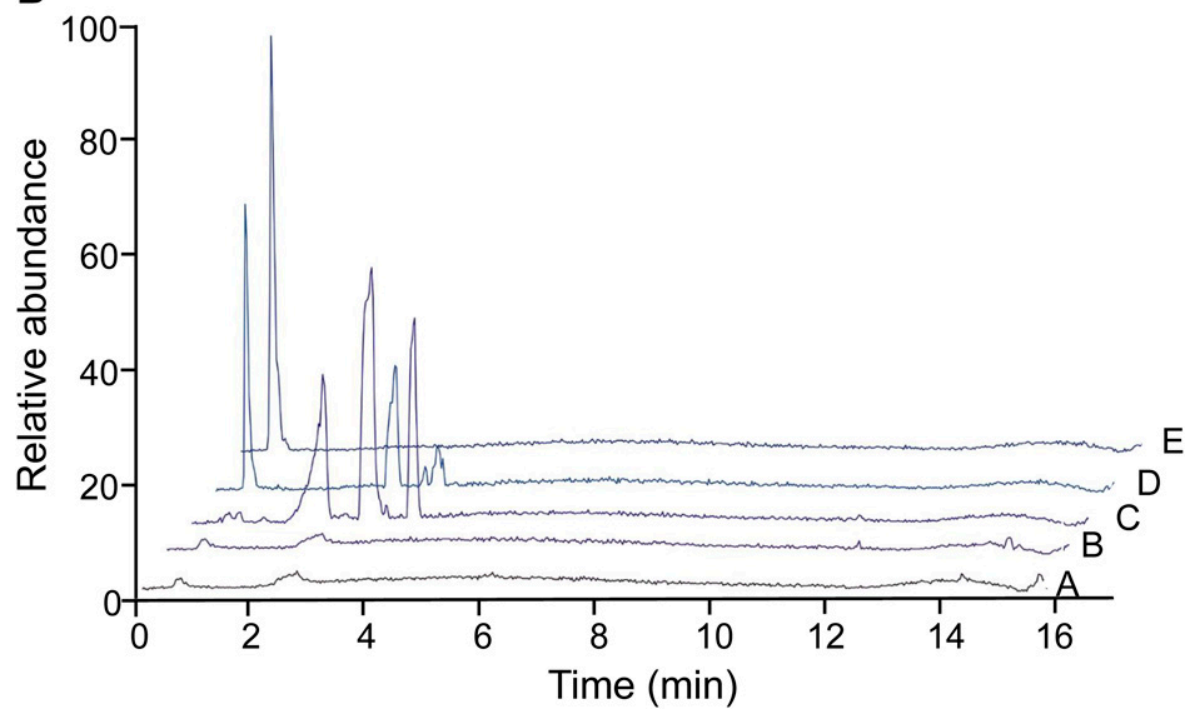

Fig. 4. (A) PLS scores plot (component 1 vs. component 2) from biochemometric analysis of four commercially available green teas (coded T02, T07, T13, and $\mathrm{T} 21)$ and the National Institute of Standards and Technology reference material (T26). (B) Stacked plot of chromatograms of fractions A-E from the T21 extract. Fractions $\mathrm{C}-\mathrm{E}$ contained the majority of peaks. Fractions D and E contained high initial solvent peaks. enterocytes (248 ml/min) (Davies and Morris, 1993). The $\mathrm{f}_{\mathrm{u}, \mathrm{g}}$ values of EGCG and ECG were set to 1 assuming no significant binding in the intestinal lumen, and $\mathrm{f}_{\mathrm{u} \text {,mic }}$ for both catechins were predicted by Simcyp to be 0.99 in HIMs at $0.05 \mathrm{mg}$ / ml. Method 2 used average simulated maximum enterocyte concentration for ECG and EGCG in duodenum, jejunum, and ileum using Simcyp. EGCG and ECG can be metabolized via glucuronidation, sulfation, and methylation, but kinetic parameters are lacking. Therefore, oral clearances from the literature were used, along with physiochemical properties, to simulate enterocyte concentrations of EGCG and ECG (Table 1). Because of the lack of experimental data and the inability to predict unbound fractions in enterocytes, the $f_{u, g}$ values of EGCG and ECG in method 2 were assumed to be the same as $f_{u, m i c}$.

\section{Results}

Biochemometrics Analysis of the First-Stage Fractionation Identified the Fraction in a Representative Green Tea Containing the Most Intestinal UGT Inhibitors. All green tea extracts and corresponding fractions (A-E) demonstrated concentration-dependent inhibition of intestinal UGT activity, as measured by 4-MU glucuronidation (Fig. 3). The effects of extracts and fractions prepared from teas T02, T07, T13, and T21 were qualitatively similar to those prepared from the National Institute of Standards and Technology reference material, whether extracted with methanol (T26) or hot water (T26-aqueous). The similarity between the methanol and hot water extraction methods was described in a previous study (Kellogg et al., 2017) in which catechins were measured in the methanol and hot water extracts prepared from the National Institute of Standards and Technology reference material. As might be expected, catechin concentrations in the hot water extract tended to be lower than those in the methanol extract, but in aggregate varied $<20 \%$.

The PLS score plot of the metabolomic profiles from the five methanolic extracts and corresponding fractions showed three distinct clusters (Fig. 4A): extract, C, and D; A and B; and E. These observations suggested that the chemical profiles of fractions $C$ and $D$ were similar to that of the extract. Based on commercial availability, sales, and consumer reports indicating frequent use, T21 (Kellogg et al., 2017) was selected for further investigation to identify candidate intestinal UGT inhibitors in green tea. Principal component analysis and a stacked plot of liquid chromatography MS base peak chromatograms corresponding to T21 fractions A-E showed that the majority of constituents were detected in fractions $\mathrm{C}-\mathrm{E}$ (Fig. 4B); fraction $\mathrm{C}$ contained the primary green tea catechins (Fig. 1) and other constituents that contributed to inhibition of intestinal 4-MU glucuronidation (Fig. 3). Because the first-stage fractionation was not sufficient to separate individual constituents from the extract, thereby precluding correlations between structure and bioactivity, fraction $\mathrm{C}$ was advanced to secondstage fractionation. The resulting subfractions $(\mathrm{C} 1-\mathrm{C} 8)$ were tested as inhibitors of intestinal UGT activity. 

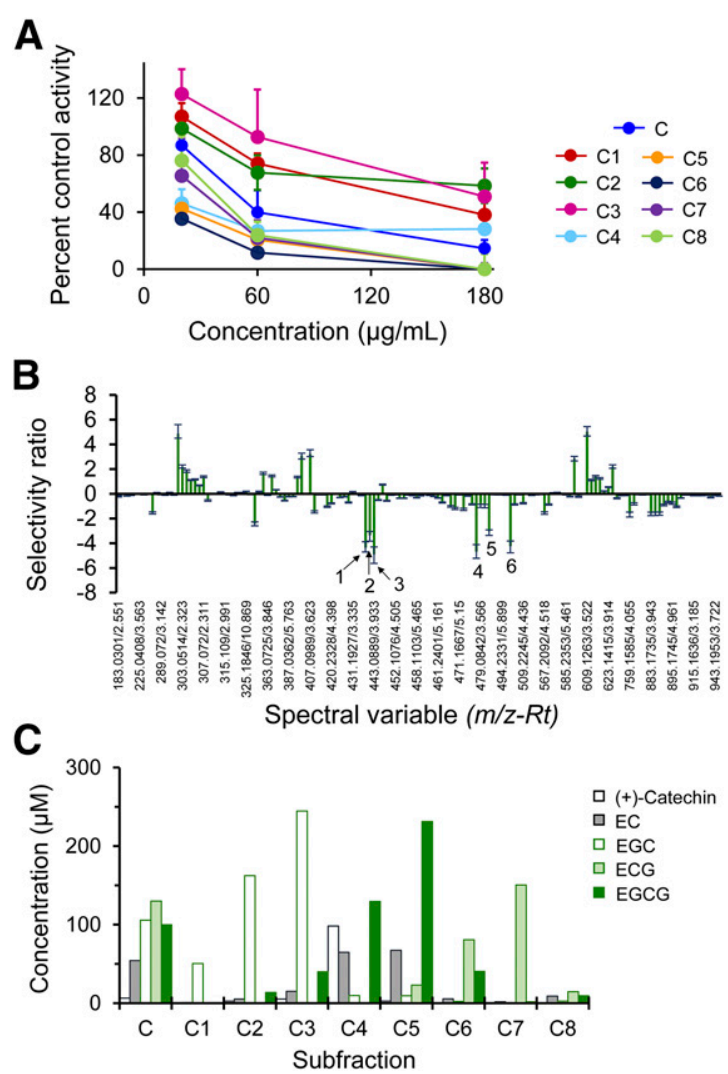

Fig. 5. (A) Concentration-dependent inhibition of intestinal microsomal UGT activity (4-MU glucuronidation) by fraction $\mathrm{C}$ and subfractions $\mathrm{C} 1-\mathrm{C} 8$ from the $\mathrm{T} 21$ extract. Symbols and error bars denote mean and S.D., respectively, of triplicate incubations. (B) Selectivity ratio analysis of the partial least-squares model data for all eight of the T21 subfractions. The more negative values represent higher contributions to the observed UGT inhibitory activity (see Table 2 for identified ions 1-6). Rt, retention time. (C) Abundance of major constituents in C1-C8 from the T21 extract based on metabolomic profiling data.

Biochemometrics Analysis of Second-Stage Fractionation Identified Five Catechins as Candidate Intestinal UGT Inhibitors. Fraction $\mathrm{C}$ and subfractions $\mathrm{C} 1-\mathrm{C} 8$ from the T21 extract generally showed concentration-dependent inhibition of 4-MU glucuronidation (Fig. 5A). An internal cross-validated PLS model was constructed from the metabolomic profiles for fraction $\mathrm{C}$ and subfractions $\mathrm{C} 1-\mathrm{C} 8$. The analysis yielded a four-component PLS model, accounting for $73.6 \%$ and $98.8 \%$ of the independent (spectral) and dependent (bioactivity) block variation, respectively. Taking the ratio between explained and residual variance of the spectral variables yielded a selectivity ratio for each marker ion (Fig. 5B). These ratios represented a quantitative measure of the contribution of each marker ion to intestinal UGT inhibitory activity. The selectivity ratio analysis highlighted six marker ions (1-6) that correlated most strongly with intestinal UGT inhibition. All marker ions eluted with the same retention time (3.93 minutes), and all were forms of the same molecule, ECG (Table 2), detected as different clusters or isotopes by the mass spectrometer. These results suggested that ECG may be the green tea constituent primarily responsible for intestinal UGT inhibitory activity. This observation was supported further by the existence of ECG in subfractions C5-C8 (Fig. 5C), which showed stronger inhibition than fraction $\mathrm{C}$ (Fig. 5A). Other catechins also were present in these fractions, including (+)-catechin, EC, EGC, and EGCG. Therefore, these five catechins were selected for further evaluation.
ECG and EGCG Are Potent Intestinal UGT Inhibitors in Green Tea. The effects of (+)-catechin, EC, EGC, ECG, and EGCG at $100 \mu \mathrm{M}$ on 4-MU glucuronidation in HIMs were compared. Only ECG and EGCG showed marked effects, inhibiting by $55 \%$ and $40 \%$ relative to vehicle control (Supplemental Fig. 1). Both ECG and EGCG showed concentration-dependent inhibition, with $\mathrm{IC}_{50}$ values of 105 and $59 \mu \mathrm{M}$, respectively (Fig. 6). The $K_{\mathrm{i}}$ values for ECG and EGCG were next determined using the clinically used intestinal UGT substrate raloxifene. The competitive inhibition model best described the data for both catechins, with $K_{\mathrm{i}}$ values of approximately 1 and $2 \mu \mathrm{M}$, respectively (Fig. 7; Supplemental Fig. 2).

A Mechanistic Static Model Predicts an Intestinal UGTMediated Green Tea/Raloxifene Interaction In Vivo. The concentrations of (+)-catechin, EC, EGC, ECG, and EGCG measured in $240 \mathrm{ml}$ of T21 tea were $7.8 \pm 1.1,95.8 \pm 3.8,285 \pm 19.1,66.4 \pm 5.0$, and $240 \pm 20.3 \mu \mathrm{M}$, respectively. Corresponding doses were $0.9 \pm 0.1$, $10.5 \pm 0.4,31.3 \pm 2.1,7.3 \pm 0.5$, and $26.4 \pm 2.2 \mathrm{mg}$, respectively. The $F_{a}$ values of ECG and EGCG were predicted by Simcyp to be 0.66 and 0.65 , respectively. Using method 1 , intestinal lumen concentrations of ECG and EGCG were predicted to be 4.4 and $15.2 \mu \mathrm{M}$, respectively. Using $K_{\mathrm{i}}$ values of 1 and $2 \mu \mathrm{M}$ for ECG and EGCG, respectively, the $\mathrm{AUC}_{\mathrm{i}} / \mathrm{AUC}$ ratios were 4.4 and 6.1, respectively. Using method 2 , the average maximum enterocyte concentrations of ECG and EGCG were estimated to be 0.18 and $0.54 \mu \mathrm{M}$, respectively, producing $\mathrm{AUC}_{\mathrm{i}} / \mathrm{AUC}$ ratios of 1.2 and 1.3 , respectively.

\section{Discussion}

Green tea is one of the most commonly consumed botanical natural products worldwide (Smith et al., 2017). These high usage patterns raise concern for co-consumption with conventional medications, prompting development of a systematic approach to evaluate potential green teadrug interactions. Compared with the cytochrome P450s and transporters, the UGTs are understudied targets for natural product-drug interactions. Like many botanical natural products, green tea contains a multitude of polyphenolic constituents that undergo extensive glucuronidation (Lu et al., 2003a; Feng, 2006). By definition, these polyphenols can act as competitive inhibitors of these enzyme(s). Because the intestine represents the first portal of entry for most drugs and other xenobiotics, intestinal UGTs could serve as key targets for natural product-drug interactions. Collectively, the goal of this work was to develop a comprehensive, robust biochemometric approach to identify and prioritize intestinal UGT inhibitors in green tea and evaluate their in vivo interaction potential. Key observations were 1) biochemometrics identified five catechins as major constituents in a selected green tea fraction as potential contributors to the UGT inhibitory effects, with ECG as a primary contributor; 2) ECG, along with EGCG, are potent inhibitors of raloxifene intestinal glucuronidation; and 3) a mechanistic static model predicted up to a 6.1-fold increase in raloxifene AUC in the presence of green tea using estimated intestinal lumen inhibitor concentrations, whereas the model predicted up to a 1.3-fold increase using estimated enterocyte inhibitor concentrations.

One challenge with bioassay-guided fractionation, a well-established method for isolating and characterizing bioactive constituents from a natural product, is identifying potential bioactive constituents for isolation among the myriad constituents in a complex extract. Biochemometrics approaches can compile both qualitative and quantitative information and prioritize constituents likely responsible for the observed bioactivity. Such an approach was recently applied to antimicrobial fungal extracts (Kellogg et al., 2016) to identify bioactive constituents after one stage of fractionation. Green tea is a more complex natural product than fungi. A total of 3607 ions were detected among the 
TABLE 2

Identities of the various forms of ECG (Fig. 5B)

All of the ions are associated with the same ECG molecule but are detected by the mass spectrometer as different isotopes or adducts.

\begin{tabular}{lcccl}
\hline Number & Ion $(\mathrm{m} / \mathrm{z})$ & Molecular Formula & $\Delta(\mathrm{ppm})$ & \multicolumn{1}{c}{ Tentative Identification } \\
\hline 1 & $443.0869[\mathrm{M}-\mathrm{H}]^{-}$ & $\mathrm{C}_{22} \mathrm{H}_{17} \mathrm{O}_{10}$ & +0.9 & ${ }^{13} \mathrm{C}$ isotope peak of ECG \\
2 & $504.0890[\mathrm{M}+\mathrm{ACN}+\mathrm{Na}-2 \mathrm{H}]^{-}$ & $\mathrm{C}_{24} \mathrm{H}_{19} \mathrm{NO}_{10} \mathrm{Na}$ & +2.7 & Acetonitrile-sodium adduct of ECG \\
3 & $477.0597[\mathrm{M}+\mathrm{Cl}]^{-}$ & $\mathrm{C}_{22} \mathrm{H}_{18} \mathrm{O}_{10} \mathrm{Cl}$ & +0.8 & Chloride adduct of ECG \\
4 & $441.0832[\mathrm{M}-\mathrm{H}]^{-}$ & $\mathrm{C}_{22} \mathrm{H}_{17} \mathrm{O}_{10}$ & +1.1 & Deprotonated molecular ion of ECG \\
5 & $442.0864[\mathrm{M}-\mathrm{H}]^{-}$ & $\mathrm{C}_{22} \mathrm{H}_{17} \mathrm{O}_{10}$ & +1.8 & ${ }^{13} \mathrm{C}$ isotope peak ECG \\
6 & $487.0886[\mathrm{M}+\mathrm{FA}-\mathrm{H}]^{-}$ & $\mathrm{C}_{23} \mathrm{H}_{19} \mathrm{O}_{12}$ & +1.0 & Formic acid adduct of ECG \\
\hline
\end{tabular}

ACN, acetonitrile; FA, formic acid.

five pooled fractions produced during the first stage of separation of a single green tea extract prepared from the reference material (T26). As such, a subfractionation procedure was necessary to adequately distribute multiple potentially active constituents among fractions (Fig. 2). This second stage of separation produced eight subfractions from the representative tea (T21), in which a total of 145 ions were detected from negative ionization mode mass spectrometric data. Many of these ions corresponded to the masses predicted for known green tea catechins. Data from the second stage biochemometric analyses demonstrated that among the 145 detected ions, those representing various forms of ECG (Table 2) were most strongly associated with UGT inhibitory activity (Fig. 5B). Thus, biochemometrics enabled identification of a bioactive catechin from among the more than 3000 ions detected.

Of the five catechins present in the T21 subfractions (Fig. 5C), only ECG and EGCG showed marked inhibition toward 4-MU glucuronidation. The $\mathrm{IC}_{50}$ values were near or below the respective concentrations measured in a cup $(240 \mathrm{ml})$ of hot tea prepared from T21 (105 and $60 \mu \mathrm{M}$ vs. 66 and $240 \mu \mathrm{M}$ ), warranting further mechanistic studies. Microplate-based fluorescence assays enable rapid measurement of enzyme activities in vitro, hence are more efficient and cost-saving compared with liquid chromatography MS/MS-based assays (Cheng et al., 2009; Kenaan et al., 2010). After demonstrating inhibition of intestinal glucuronidation with this method (Gufford et al., 2014), subsequent experiments were conducted with the clinically used UGT substrate, raloxifene. Raloxifene, which undergoes extensive intestinal glucuronidation (oral bioavailability $\sim 2 \%$ ), was used to determine the $K_{\mathrm{i}}$ values for ECG and EGCG. Both were potent competitive inhibitors, with $K_{\mathrm{i}}$ values of $\sim 1$ and $2 \mu \mathrm{M}$, respectively. Assuming ECG and EGCG also inhibit 4-MU glucuronidation in a competitive manner (i.e., $\mathrm{IC}_{50} \sim 2 K_{\mathrm{i}}$ ) (Cheng and Prusoff, 1973), these $K_{\mathrm{i}}$ values were less than one-tenth the corresponding values using 4-MU. 4-MU is glucuronidated by multiple UGTs, including several from both the UGT1A and UGT2B families (Uchaipichat et al., 2004), whereas raloxifene is glucuronidated by UG1A1, $-1 \mathrm{~A} 8$, and $-1 \mathrm{~A} 10$, the latter two of which are expressed in the intestine but not the liver (Wu et al., 2011). Taken together, the higher inhibitory potency of ECG and EGCG toward raloxifene glucuronidation in HIMs may be due to inhibition of specific intestinal UGTs, such as UGT1A8 and UGT1A10.

The application of a mechanistic static model to predict the magnitude of a drug-drug interaction in vivo $\left(\mathrm{AUC}_{\mathrm{i}} / \mathrm{AUC}\right)$ relies on a robust estimate of the inhibitor concentration (Obach et al., 2006). Regarding intestinal enzyme-mediated interactions, the most appropriate inhibitor concentration would be that available to intestinal enzymes. Accordingly, two methods were used to estimate intestinal inhibitor concentrations. Method 1, which does not consider metabolism of the inhibitor, often overpredicts the interaction potential (Gufford et al., 2015a,b). Method 2, which considers metabolism of the inhibitor and simulates inhibitor concentrations within enterocytes, may provide a more reasonable prediction. These simulated concentrations were then applied to a mechanistic static model, which was subsequently used as a decision tool about whether to develop a dynamic model that describes the whole system. Application of method 1 to the $\mathrm{AUC}_{\mathrm{i}} / \mathrm{AUC}$ ratio calculation led to a 4.4- to 6.1-fold increase, whereas method 2 led to a 1.2- to 1.3-fold increase, in raloxifene plasma AUC. Despite the discrepant predictions, these results support further evaluation of green tea as an inhibitor of intestinal UGT in vivo via dynamic modeling approaches and clinical testing (Gufford et al., 2015b).

There are limitations to the current work. First, only fraction $\mathrm{C}$ from T21 was advanced to second-stage fractionation. Other fractions also demonstrated UGT inhibitory activity (Fig. 3), and follow-up studies (similar to those presented in the current work) would be needed to identify potential inhibitors in those fractions. Thus, although the current work suggests catechins are contributors to the in vitro UGT inhibitory activity of green tea extracts, other as yet unidentified inhibitors are present in the complex extract. Second, although ECG and EGCG were identified and characterized as potent intestinal UGT inhibitors, they may not represent the overall effect of green tea fraction $C$. Testing of the three other identified catechins [(+)-catechin, EC, and EGC] showed weak inhibition $(20 \%-50 \%)$ of raloxifene glucuronidation at $100 \mu \mathrm{M}$ (Supplemental Fig. 3); because these catechins are typically abundant in
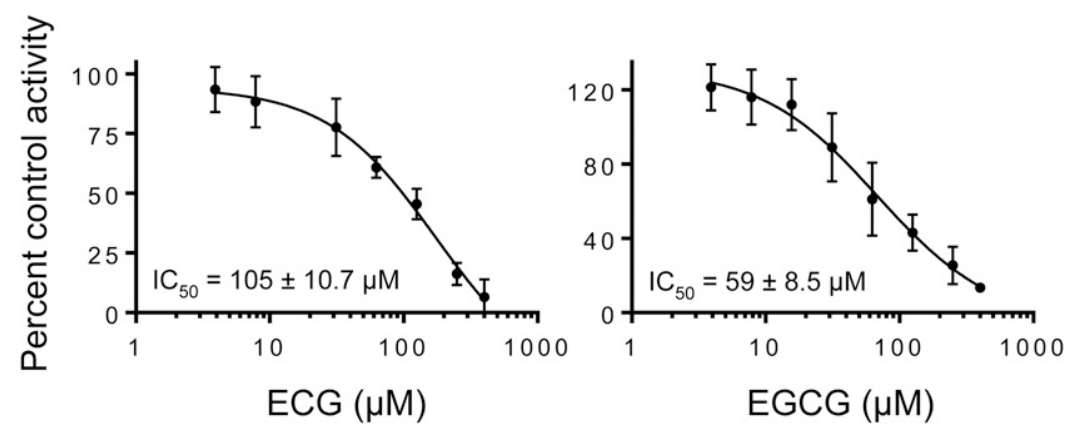

Fig. 6. Concentration-dependent inhibition of intestinal microsomal UGT activity (4-MU glucuronidation) by ECG and EGCG. Symbols and error bars denote mean and S.D., respectively, of triplicate incubations. Curves denote nonlinear least-squares regression of the data. 
R4G

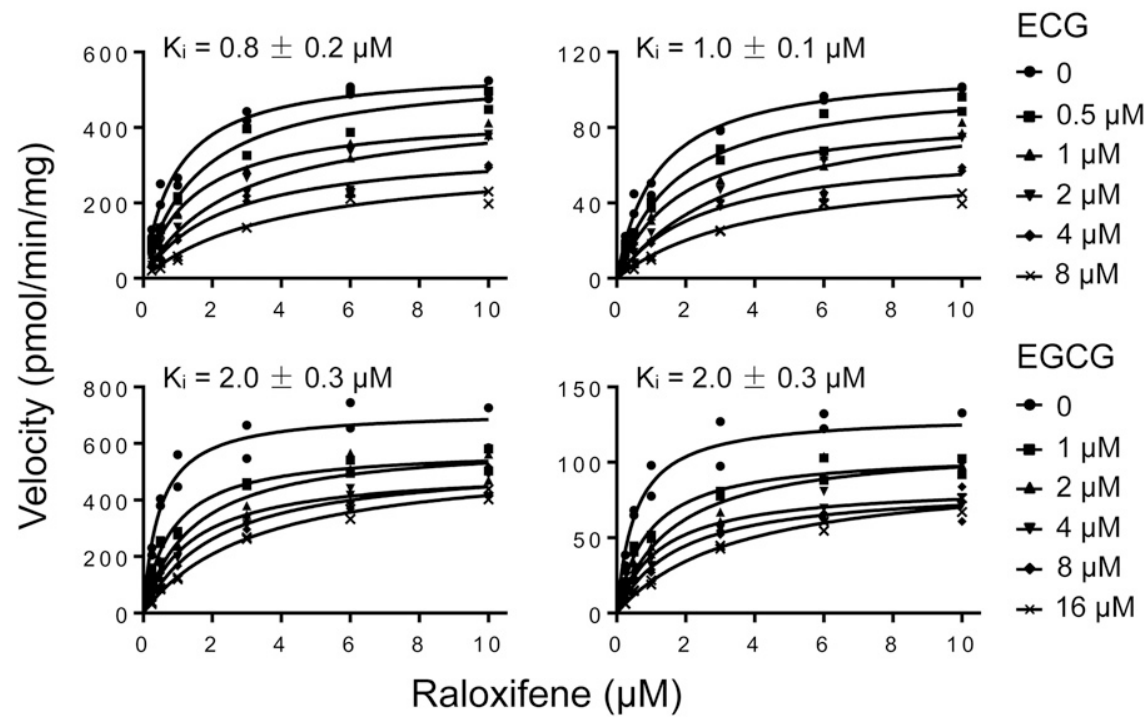

Fig. 7. Kinetics of inhibition of raloxifene-4' -glucuronide (R4G; left) or raloxifene-6-glucuronide (R6G; right) formation by ECG (upper) and EGCG (lower). Symbols denote individual data points of duplicate incubations. Velocity vs. substrate concentration data were described best by the simple competitive inhibition model. Curves denote nonlinear least-squares regression of the data. green tea, they may contribute to the overall effect of the tea. In addition, physical interactions (e.g., complexation) and/or biochemical interactions (e.g., additivity, synergy, and antagonism) between the catechins or other constituents could contribute to the overall effect of the tea, studies of which were beyond the scope of this study. Third, use of a fluorescent microplate assay with the pan UGT substrate 4-MU, although cost effective, may have missed weak inhibitors. However, the goal of this work was to identify strong (rather than all possible) intestinal UGT inhibitors, with the long-range goal of identifying clinically relevant green tea-drug interactions. This high-throughput assay also could be applied to UGTs expressed in the liver and kidney, as well as to other natural products.

In summary, identification of bioactive constituents, including inhibitors of drug metabolizing enzymes, in complex botanical products requires a comprehensive, multi-disciplinary approach. The biochemometrics approach described in the current work was highly effective in identifying candidate intestinal UGT inhibitors in a selected green tea fraction and prioritizing these inhibitors for further evaluation. Application of the most potent inhibitors to a mechanistic static model suggested that green tea could precipitate a clinical interaction with raloxifene and potentially other clinically used intestinal UGT substrates (e.g., ezetimibe and mycophenolic acid). This identification and prioritization process could be applied to other green tea fractions to identify additional intestinal UGT inhibitors, as well as inhibitors of other interaction targets, including additional drug metabolizing enzymes and transporters. As regulatory guidance for drug-drug interactions continues to evolve, the pharmaceutical industry may in the near future need to adopt such an approach to determine the drug interaction liability of a given natural product toward a new molecular entity. Application of this biochemometric approach to other natural products will refine the procedures and contribute to decision trees (European Medicines Agency, 2012; Food and Drug Administration Center for Drug Evaluation and Research, 2017).

\section{Acknowledgments}

Mass spectrometry data were collected at the Triad Mass Spectrometry Facility at the University of North Carolina at Greensboro. M.F.P. dedicates this article to Dr. David P. Paine.

\section{Authorship Contributions}

Participated in research design: Tian, Kellogg, Oberlies, Cech, Shen, McCune, Paine.

Conducted experiments: Tian, Kellogg, Okut.

Contributed new reagents or analytic tools: Kellogg, Okut, Oberlies, Cech. Performed data analysis: Tian, Kellogg, Cech, Paine.

Wrote or contributed to the writing of the manuscript: Tian, Kellogg, Oberlies, Cech, Shen, McCune, Paine.

\section{References}

Balentine DA, Wiseman SA, and Bouwens LC (1997) The chemistry of tea flavonoids. Crit Rev Food Sci Nutr 37:693-704.

Brantley SJ, Argikar AA, Lin YS, Nagar S, and Paine MF (2014) Herb-drug interactions: challenges and opportunities for improved predictions. Drug Metab Dispos 42:301-317.

Britton ER, Kellogg JJ, Kvalheim OM, and Cech NB (2017) Biochemometrics to identify synergists and additives from botanical medicines: a case study with Hydrastis canadensis (goldenseal). J Nat Prod DOI: 10.1021/acs.jnatprod.7b00654 [published ahead of print].

Cheng Q, Sohl CD, and Guengerich FP (2009) High-throughput fluorescence assay of cytochrome P450 3A4. Nat Protoc 4:1258-1261.

Cheng Y and Prusoff WH (1973) Relationship between the inhibition constant $\left(K_{I}\right)$ and the concentration of inhibitor which causes 50 per cent inhibition $\left(I_{50}\right)$ of an enzymatic reaction. Biochem Pharmacol 22:3099-3108.

Chow HH, Cai Y, Alberts DS, Hakim I, Dorr R, Shahi F, Crowell JA, Yang CS, and Hara Y (2001) Phase I pharmacokinetic study of tea polyphenols following single-dose administration of epigallocatechin gallate and polyphenon E. Cancer Epidemiol Biomarkers Prev 10:53-58.

Chow HH, Cai Y, Hakim IA, Crowell JA, Shahi F, Brooks CA, Dorr RT, Hara Y, and Alberts DS (2003) Pharmacokinetics and safety of green tea polyphenols after multiple-dose administration of epigallocatechin gallate and polyphenon $\mathrm{E}$ in healthy individuals. Clin Cancer Res 9: $3312-3319$.

Chow HH, Hakim IA, Vining DR, Crowell JA, Cordova CA, Chew WM, Xu MJ, Hsu CH, RangerMoore J, and Alberts DS (2006) Effects of repeated green tea catechin administration on human cytochrome P450 activity. Cancer Epidemiol Biomarkers Prev 15:2473-2476.

Cubitt HE, Houston JB, and Galetin A (2011) Prediction of human drug clearance by multiple metabolic pathways: integration of hepatic and intestinal microsomal and cytosolic data. Drug Metab Dispos 39:864-873.

Davies B and Morris T (1993) Physiological parameters in laboratory animals and humans. Pharm Res 10:1093-1095.

Deka A and Vita JA (2011) Tea and cardiovascular disease. Pharmacol Res 64:136-145.

Donovan JL, Chavin KD, Devane CL, Taylor RM, Wang JS, Ruan Y, and Markowitz JS (2004) Green tea (Camellia sinensis) extract does not alter cytochrome P450 3A4 or 2D6 activity in healthy volunteers. Drug Metab Dispos 32:906-908.

Drozdzik M, Gröer C, Penski J, Lapczuk J, Ostrowski M, Lai Y, Prasad B, Unadkat JD, Siegmund W, and Oswald S (2014) Protein abundance of clinically relevant multidrug transporters along the entire length of the human intestine. Mol Pharm 11:3547-3555.

European Medicines Agency (2012) Guideline on the Investigation of Drug Interactions, European Medicines Agency, London.

Fahmi OA, Hurst S, Plowchalk D, Cook J, Guo F, Youdim K, Dickins M, Phipps A, Darekar A, Hyland R, et al. (2009) Comparison of different algorithms for predicting clinical drug-drug interactions, based on the use of CYP3A4 in vitro data: predictions of compounds as precipitants of interaction. Drug Metab Dispos 37:1658-1666.

Feng WY (2006) Metabolism of green tea catechins: an overview. Curr Drug Metab 7:755-809. 
Food and Drug Administration Center for Drug Evaluation and Research (2013) Bioanalytical Method Validation (Draft Guidance), U.S. Food and Drug Administration, Silver Spring, MD. Food and Drug Administration Center for Drug Evaluation and Research (2017) In Vitro Metab olism and Transporter Mediated Drug-Drug Interaction Studies Guidance for Industry (Draft Guidance), U.S. Food and Drug Administration, Silver Spring, MD.

Glaeser H, Bailey DG, Dresser GK, Gregor JC, Schwarz UI, McGrath JS, Jolicoeur E, Lee W, Leake BF, Tirona RG, et al. (2007) Intestinal drug transporter expression and the impact of grapefruit juice in humans. Clin Pharmacol Ther 81:362-370.

Graf TN, Wani MC, Agarwal R, Kroll DJ, and Oberlies NH (2007) Gram-scale purification of flavonolignan diastereoisomers from Silybum marianum (milk thistle) extract in support of preclinical in vivo studies for prostate cancer chemoprevention. Planta Med 73:1495-1501.

Gufford BT, Barr JT, González-Pérez V, Layton ME, White JR, Jr, Oberlies NH, and Paine MF (2015a) Quantitative prediction and clinical evaluation of an unexplored herb-drug interaction mechanism in healthy volunteers. CPT Pharmacometrics Syst Pharmacol 4:701-710.

Gufford BT, Chen G, Lazarus P, Graf TN, Oberlies NH, and Paine MF (2014) Identification of diet-derived constituents as potent inhibitors of intestinal glucuronidation. Drug Metab Dispos 42:1675-1683.

Gufford BT, Chen G, Vergara AG, Lazarus P, Oberlies NH, and Paine MF (2015b) Milk thistle constituents inhibit raloxifene intestinal glucuronidation: a potential clinically relevant natural product-drug interaction. Drug Metab Dispos 43:1353-1359.

Inui T, Wang Y, Pro SM, Franzblau SG, and Pauli GF (2012) Unbiased evaluation of bioactive secondary metabolites in complex matrices. Fitoterapia 83:1218-1225.

Ito K, Iwatsubo T, Kanamitsu S, Ueda K, Suzuki H, and Sugiyama Y (1998) Prediction of pharmacokinetic alterations caused by drug-drug interactions: metabolic interaction in the liver. Pharmacol Rev 50:387-412.

Jenkinson C, Petroczi A, Barker J, and Naughton DP (2012) Dietary green and white teas suppress UDP-glucuronosyltransferase UGT2B17 mediated testosterone glucuronidation. Steroids 77 691-695.

Kellogg JJ, Graf TN, Paine MF, McCune JS, Kvalheim OM, Oberlies NH, and Cech NB (2017) Comparison of metabolomics approaches for evaluating the variability of complex botanical preparations: green tea (Camellia sinensis) as a case study. J Nat Prod 80:1457-1466.

Kellogg JJ, Todd DA, Egan JM, Raja HA, Oberlies NH, Kvalheim OM, and Cech NB (2016) Biochemometrics for natural products research: comparison of data analysis approaches and application to identification of bioactive compounds. J Nat Prod 79:376-386.

Kenaan C, Zhang H, and Hollenberg PF (2010) A quantitative high-throughput 96-well plate fluorescence assay for mechanism-based inactivators of cytochromes P450 exemplified using CYP2B6. Nat Protoc 5:1652-1658.

Kim E, Sy-Cordero A, Graf TN, Brantley SJ, Paine MF, and Oberlies NH (2011) Isolation and identification of intestinal CYP3A inhibitors from cranberry (Vaccinium macrocarpon) using human intestinal microsomes. Planta Med 77:265-270.

Knop J, Misaka S, Singer K, Hoier E, Müller F, Glaeser H, König J, and Fromm MF (2015) Inhibitory effects of green tea and (-)-epigallocatechin gallate on transport by OATP1B1, OATP1B3, OCT1, OCT2, MATE1, MATE2-K and P-glycoprotein. PLoS One 10:e139370.

Kvalheim OM, Chan HY, Benzie IFF, Szeto YT, Tzang AHC, Mok DKW, and Chau FT (2011) Chromatographic profiling and multivariate analysis for screening and quantifying the contributions from individual components to the bioactive signature in natural products. Chemometr Intell Lab Syst 107:98-105.

Lu H, Meng X, Li C, Sang S, Patten C, Sheng S, Hong J, Bai N, Winnik B, Ho CT, et al. (2003a) Glucuronides of tea catechins: enzymology of biosynthesis and biological activities. Drug Metab Dispos 31:452-461

Lu H, Meng X, and Yang CS (2003b) Enzymology of methylation of tea catechins and inhibition of catechol-O-methyltransferase by (-)-epigallocatechin gallate. Drug Metab Dispos 31: 572-579.

Misaka S, Kawabe K, Onoue S, Werba JP, Giroli M, Tamaki S, Kan T, Kimura J, Watanabe H, and Yamada S (2013) Effects of green tea catechins on cytochrome P450 2B6, 2C8, 2C19, 2D6 and $3 \mathrm{~A}$ activities in human liver and intestinal microsomes. Drug Metab Pharmacokinet 28 : 244-249.

Misaka S, Knop J, Singer K, Hoier E, Keiser M, Müller F, Glaeser H, König J, and Fromm MF (2016) The nonmetabolized $\beta$-blocker nadolol is a substrate of OCT1, OCT2, MATE1, MATE2 K, and P-glycoprotein, but not of OATP1B1 and OATP1B3. Mol Pharm 13:512-519.

Misaka S, Yatabe J, Müller F, Takano K, Kawabe K, Glaeser H, Yatabe MS, Onoue S, Werba JP, Watanabe H, et al. (2014) Green tea ingestion greatly reduces plasma concentrations of nadolol in healthy subjects. Clin Pharmacol Ther 95:432-438.

Mizuma T (2009) Intestinal glucuronidation metabolism may have a greater impact on oral bioavailability than hepatic glucuronidation metabolism in humans: a study with raloxifene, substrate for UGT1A1, 1A8, 1A9, and 1A10. Int J Pharm 378:140-141.
Mohamed ME and Frye RF (2011) Inhibitory effects of commonly used herbal extracts on UDPglucuronosyltransferase 1A4, 1A6, and 1A9 enzyme activities. Drug Metab Dispos 39: $1522-1528$.

Mohamed MF, Tseng T, and Frye RF (2010) Inhibitory effects of commonly used herbal extracts on UGT1A1 enzyme activity. Xenobiotica 40:663-669.

Mooiman KD, Goey AK, Huijbregts TJ, Maas-Bakker RF, Beijnen JH, Schellens JH, and Meijerman I (2014) The in-vitro effect of complementary and alternative medicines on cytochrome P450 2C9 activity. J Pharm Pharmacol 66:1339-1346.

Moore RJ, Jackson KG, and Minihane AM (2009) Green tea (Camellia sinensis) catechins and vascular function. Br J Nutr 102:1790-1802.

Muto S, Fujita K, Yamazaki Y, and Kamataki T (2001) Inhibition by green tea catechins of metabolic activation of procarcinogens by human cytochrome P450. Mutat Res 479:197-206.

Nakagawa K, Okuda S, and Miyazawa T (1997) Dose-dependent incorporation of tea catechins, (-)-epigallocatechin-3-gallate and (-)-epigallocatechin, into human plasma. Biosci Biotechnol Biochem 61:1981-1985.

Ngo N, Yan Z, Graf TN, Carrizosa DR, Kashuba AD, Dees EC, Oberlies NH, and Paine MF (2009) Identification of a cranberry juice product that inhibits enteric CYP3A-mediated first-pass metabolism in humans. Drug Metab Dispos 37:514-522.

Obach RS, Walsky RL, Venkatakrishnan K, Gaman EA, Houston JB, and Tremaine LM (2006) The utility of in vitro cytochrome $\mathrm{P} 450$ inhibition data in the prediction of drug-drug interactions. I Pharmacol Exp Ther 316:336-348.

Pluskal T, Castillo S, Villar-Briones A, and Oresic M (2010) MZmine 2: modular framework for processing, visualizing, and analyzing mass spectrometry-based molecular profile data. BMC Bioinformatics 11:395.

Renouf M, Marmet C, Guy PA, Beaumont M, Lepage M, Williamson G, and Dionisi F (2013) Dose-response plasma appearance of green tea catechins in adults. Mol Nutr Food Res 57: 833-839.

Rostami-Hodjegan A and Tucker G (2004) 'In silico' simulations to assess the 'in vivo' consequences of 'in vitro' metabolic drug-drug interactions. Drug Discov Today Technol 1:441-448.

Roth M, Araya JJ, Timmermann BN, and Hagenbuch B (2011) Isolation of modulators of the liverspecific organic anion-transporting polypeptides (OATPs) 1B1 and 1B3 from Rollinia emarginata Schlecht (Annonaceae). J Pharmacol Exp Ther 339:624-632.

Sang S, Lambert JD, Ho CT, and Yang CS (2011) The chemistry and biotransformation of tea constituents. Pharmacol Res 64:87-99.

Schönthal AH (2011) Adverse effects of concentrated green tea extracts. Mol Nutr Food Res 55: 874-885.

Smith T, Kawa K, Eckl V, Morton C, and Stredney R (2017) Herbal supplement sales in US increase $7.7 \%$ in 2016: consumer preferences shifting toward ingredients with general wellness benefits, driving growth of adaptogens and digestive health products. HerbalGram 115:56-65.

Song Q, Li D, Zhou Y, Yang J, Yang W, Zhou G, and Wen J (2014) Enhanced uptake and transport of (+)-catechin and (-)-epigallocatechin gallate in niosomal formulation by human intestinal Caco-2 cells. Int J Nanomedicine 9:2157-2165.

Uchaipichat V, Mackenzie PI, Guo XH, Gardner-Stephen D, Galetin A, Houston JB, and Miners JO (2004) Human UDP-glucuronosyltransferases: isoform selectivity and kinetics of 4-methylumbelliferone and 1-naphthol glucuronidation, effects of organic solvents, and inhibition by diclofenac and probenecid. Drug Metab Dispos 32:413-423.

Vaidyanathan JB and Walle T (2003) Cellular uptake and efflux of the tea flavonoid (-)epicatechin3-gallate in the human intestinal cell line Caco-2. J Pharmacol Exp Ther 307:745-752.

Won CS, Oberlies NH, and Paine MF (2012) Mechanisms underlying food-drug interactions: inhibition of intestinal metabolism and transport. Pharmacol Ther 136:186-201.

Wu B, Kulkarni K, Basu S, Zhang S, and Hu M (2011) First-pass metabolism via UDPglucuronosyltransferase: a barrier to oral bioavailability of phenolics. J Pharm Sci 100: $3655-3681$.

Yang CS and Pan E (2012) The effects of green tea polyphenols on drug metabolism. Expert Opin Drug Metab Toxicol 8:677-689.

Yang CS and Wang H (2011) Mechanistic issues concerning cancer prevention by tea catechins. Mol Nutr Food Res 55:819-831.

Yu J, Zhou Z, Tay-Sontheimer J, Levy RH, and Ragueneau-Majlessi I (2017) Intestinal drug interactions mediated by OATPs: a systematic review of preclinical and clinical findings. $J$ Pharm Sci 106:2312-2325.

Address correspondence to: Dr. Mary F. Paine, Department of Pharmaceutical Sciences, College of Pharmacy, Washington State University, PBS 341, P.O. Box 1495, Spokane, WA 99210-1495. E-mail: mary.paine@wsu.edu 